\title{
Costumbres en torno al parto en la comunidad nativa Camisea del pueblo Matsigenka, Cusco, Perú
}

La introducción de la medicina occidental a través de programas de salud pública y la instalación de centros médicos en muchas de las regiones del país, y en especial en regiones rurales, han generado desencuentros entre las prácticas tradicionales locales enfocadas en cómo dar a luz; generando una desvalorización en las prácticas culturales para el cuidado del embarazo y parto y el desprestigio de las y los curanderos y parteras. El presente artículo se propone describir las costumbres relacionadas al parto que actualmente las mujeres del pueblo Matsigenka en la comunidad nativa de Camisea practican. De esta manera comprender las concepciones y costumbres que las mujeres tienen sobre sus partos, además de los aprendizajes durante la primera menstruación, los cuidados durante el periodo del embarazo y posparto; finalmente explicando la relación existente con el sistema de salud público.

PALABRAS ClAVE: Matsigenka, parto, prácticas tradicionales, partera, Perú.

\section{Customs related to childbirth in the Matsigenka indigenous community of Camisea, Cusco, Peru}

\begin{abstract}
The introduction of Western medicine through public health programs and new medical centers in many regions, especially in rural areas, has generated conflict between local traditional practitioners and Western modes of care, specifically around pregnancy and childbirth. This in turn, has generated a general devaluing of traditional practices of healthcare, and the degradation and discrediting of traditional healers and midwives. This article aims to describe the customs related to childbirth that Matsigenka women in the native community of Camisea currently practice. Additionally, this article looks at what is taught to a young woman during her first menstruation, care during the pregnancy and postpartum periods; finally explains the relationship of the Matisgenka women of the Camisea community with the public health system.
\end{abstract}

KEYWORDS: Matsigenka, childbirth, traditional practices, midwife, Peru.

\footnotetext{
Proyecto de Investigación de la Unidad de Posgrado de la Facultad de Ciencias Sociales de la Universidad Nacional Mayor de San Marcos. Nacer Matsigenka: Prácticas tradicionales en torno al parto en la comunidad nativa de Camisea. Resolución Mg.CC.SS-PS.GP-139 / 03.11.2015. Asesora: María Emma Mannarelli Cavagnari.
} 


\section{Introducción}

$\square$ parto es un evento biológico y cultural que da inicio a la vida y las relaciones en un grupo social. Según la cultura a la que se pertenece existen prácticas, creencias y costumbres en torno al parto (Zevallos, 2010: 115).

Es común, que después del primer parto, las mujeres puedan parir solas o acompañadas de sus familiares. El parto se da en un ámbito femenino, se puede observar a nivel comunal la presencia de mujeres que desarrollan un mayor número de conocimientos y prácticas para atender a las embarazadas en sus primeros partos o con dificultades, ellas son conocidas como parteras tradicionales.

Si bien existen estudios realizados sobre los aspectos socioculturales y necesidades de la salud reproductiva en comunidades indígenas amazónicas ${ }^{1}$, el tema de la interculturalidad en salud ha recibido en Perú muy escasa atención de las ciencias sociales (Nureña, 2009: 375).

Este tipo de estudios permiten mejorar las propuestas en las políticas públicas en salud, como es el caso para salud reproductiva, la actualizada «Norma Técnica de salud para la atención del parto vertical en el marco de los derechos humanos con pertinencia intercultural» (Norma Técnica de Salud $518,2016)$, la cual ha obtenido algunas experiencias exitosas en población rural andina (Salud Sin Límites Perú, 2009).

El ámbito de la presente investigación es la comunidad nativa de Camisea, perteneciente al pueblo Matsigenka, ubicada en la cuenca del Bajo Urubamba. Es una comunidad representativa de los cambios socioculturales acelerados que vive la población indígena amazónica. Se encuentra próxima al proyecto gasífero más importante del país ${ }^{2}$. En ella, como en cualquier otro lugar, el parto es un evento de importancia para la construcción social y cultural en su conjunto.

Con el objetivo de conocer y analizar las costumbres y prácticas culturales relacionadas a la atención del parto de las mujeres de la comunidad nativa de Camisea del pueblo Matsigenka del Bajo Urubamba se

1 Bant y Motta, 2001; CARE-Perú, 2002; Fuller, 2009; Medina y Mayca, 2006; Ministerio de Salud, 2000, 1999; Rivas, 1994; Salud Sin Límites Perú, 2008, 2009; Vargas y Naccarato, 1995; Velarde, 2010; Villavicencio, 2002; entre otros.

2 La empresa Pluspetrol empieza sus actividades de explotación en el Lote 88 a partir del ańo 2000 (Decreto Supremo No 021-2000-EM). determinó las preferencias para parir de las mujeres de la comunidad nativa Camisea del pueblo Matsigenka del Bajo Urubamba, se analizó las diferencias existentes entre el parto en casa tradicional y el parto en el centro de salud en la comunidad nativa y se registró los recursos locales (plantas, animales, minerales) empleados en el embarazo, parto y postparto por las mujeres de la comunidad nativa Camisea del pueblo Matsigenka del Bajo Urubamba.

El estudio etnográfico se dirigió a conocer la concepción, costumbres y prácticas en torno al parto de las mujeres del pueblo Matsigenka, también se consideró de importancia el registro de los recursos locales (plantas, animales, minerales u otros elementos) utilizados por las mujeres durante la gestación, parto y posparto.

Las herramientas empleadas fueron principalmente la realización de entrevistas individuales a profundidad, observación participante, la conducción de dos talleres con las obstetras del centro de salud de Camisea y la revisión de documentos etnográficos. Para las entrevistadas se seleccionaron a mujeres en edad reproductiva, hombres adultos mayores, parteras, personal de salud (biólogo, obstetras, técnico sanitario, enfermera técnica). Adicionalmente se entabló conversaciones con mujeres, hombres, parteras, y personal de la microred de Camisea, pertenecientes a otras comunidades.

Asimismo, se tuvo la participación de una joven madre perteneciente a Camisea, Yaquemilsa Matiashi Vicente, quien participó en el registro de los datos etnográficos de las mujeres mayores que en su mayoría solo se comunican en su idioma materno. Además, al finalizar la investigación elaboró dibujos representando las creencias y costumbres relacionadas parto del pueblo Matsigenka con el fin facilitar la visualización de las mismas al personal de salud y otros agentes de salud en próximas publicaciones.

\section{La primera menstruación}

Siendo entendida la primera menstruación como una transición de nińas a mujeres, las mujeres del pueblo Matsigenka tienen que vivir un periodo en donde se realizan dietas para adecuar el cuerpo, a los nuevos conocimientos y eventos de la vida adulta. Un aprendizaje que las aísla de la familia a excepción de la mujer que las orienta. Si bien en las comunidades 
más alejadas de la cuenca del bajo Urubamba se siguen llevando a cabo las dietas estrictas y la reclusión prolongada, en la comunidad de Camisea también, aunque la duración es menor.

Existen restricciones alimenticias y de comportamiento que las mujeres deben de considerar, sobre todo en la primera menstruación, para no tener luego problemas de salud o de sus familiares. Durante este periodo su alimentación se basaba en yuca sancochada, en ocasiones acompañado de un pescado pequeño como carachama o chiripia ${ }^{3}$; sumado a comidas especiales como la patarashca ${ }^{4}$ de tsiriniro, animal parecido a una anguila, que sirve también para que el parto sea fácil y las mujeres no sufran mucho dolor 5 .

Era prohibido el consumo de sal o alimentos dulces por provocar caries causando la pérdida de dientes, de mayores no les durarían los dientes (Izquierdo, 2001: 125). Lo mismo era con los animales que tenían dientes (incluido los peces), ni animales que tengan mucha sangre como la sachavaca, sajino, o que se cocinaran empacados (forma de asar la carne empleando cańas como envoltorio), estaba negado comerlos porque cuando se fuera madre podía ocasionar que se tenga muchos hijos continuamente y la sangre aumentara mucho más de lo que fue en su primera menstruación.

Comer carne de mono durante la primera menstruación o en los embarazos podría provocar manchas en la cara de la mujer; la carne de venado las haría mujeres que corretean; si comieran carne de suri serían chismosas (Vela, 2011). Una de las entrevistadas informó que no se pueden comer larvas que contengan dientes como el suri, chagavento, kao, tsuro, entre otros. Según dicen serían mordidas por dentro y sangrarían.

Habían prohibiciones con respecto a la Luna, si se la miraba, podía ennegrecerles la cara, acecharles en la noche para matarlas, «la luna es la cabeza humana manchada con la sangre de la mujer». Señalarla con el dedo podría ocasionar el perderlo.

3 Podían comer pescado pero evitando la cabeza y cola. Vela (2011), informa que también es permitido comer caracolitos de río y la palmera chonta.

4 Se le dice al asado o hervido de cualquier carne o también hongos y fibras de plantas en el que se emplea hojas de bijao para envolver. Esta planta le da un sabor muy agradable a la comida.

5 Este animal también es empleado en el primer mes de embarazo. Si se llega a pescar se toma al animal para sobarlo en la espalda de la mujer y luego soltarlo al río, de esta manera el bebé nace como resbalando.
Dice porque la luna te puede echar su... será pues qué cosa, te puedes volverte negra... dice que la luna te puede echar agüita, qué será pero la costumbre... qué será pero la cosa es no te dejan mirar. Si por eso a las 3 de la mañana te sacan a bañar a esa hora, todo oscuro... (R. Semperi, entrevista, 26-10-2016).

Si por motivos de ir al baño salían de la reclusión, lo hacían tapadas y sin ver el cielo y los alrededores; caso contrario ocasionaría que se quedaran morenas o atraer a los espíritus del bosque y estos hacerles daño.

Las mujeres mayores comentaron que su aislamiento se realizaba en pequeñas chozas de estera elaborada con hojas de palmera, eran encerradas entre una a tres lunas, es decir alrededor de tres meses. Sólo una de ellas informó que estuvo cerca de un año. En comparación a las mujeres jóvenes, la mayoría fueron encerradas una semana, algunas un mes, aunque cuando se da la participación de las abuelas, es más tiempo.

\section{Aprendizajes durante la menarquía}

Como parte de los aprendizajes durante la primera menstruación las madres les prohibían hablar para aprender de mayores a no hablar gritando porque se dice que luego serían escandalosas. Siempre eran aconsejadas, por las mujeres mayores de la familia, durante las visitas diarias mientras eran bañadas con agua caliente para quitarles la suciedad de la niñez.

Aunque la primera menstruación se la recuerda como un momento de sus vidas que sufrieron recluidas estando solas, a oscuras y obligadas a dietas estrictas, también lo recuerdan como un momento de aprendizaje.

Al estar sola en lo oscuro ahí aprendes a pensar y soñar muchas cosas. Aprendes como trabajar hilando para no tener frio durante la época de lluvia (Shina, entrevista, 06-12-2014).

\section{Prácticas para renovar el cuerpo en la menarquía}

Como otros autores mencionan (Izquierdo, 2001; Izquierdo y Shepard, 2003; Barrieles, 1977 citado en Encinas, 2008) durante la primera menstruación se dan bańos con agua caliente. Para conocer mejor sobre la manera de estos baños se le consultó a la enfermera 
técnica Matsigenka de la comunidad de Timpía, Rita Semperi, es así como se evidencian los masajes especiales para acomodar el cuerpo con el fin de la maternidad.

$\mathrm{Si}$, te empieza agarrarte, te masajean así para abajo todo tu barriga te lo masajean para abajo. Te dicen que es para que estés bien preparada y que no sufras al momento de dar a luz y cuando tengas tu bebito. $\mathrm{Y}$ te masajean toda la espalda para abajo, nunca para arriba, sino para abajo... de ahí te ponen...te amarran tu barriga... de ahí te encierran un mes, dos meses de luna. De ahí te bañas a las 3 de la madrugada. De ahí te sacan y de nuevo te amarran (R. Semperi, entrevista, 26-10-2016).

Al salir de la reclusión también se las bañaba con agua muy caliente, con la intención de adquirir fortaleza privándoles el poder expresar alguna incomodidad; se las pintaba con huito ${ }^{6}$; tomaban purgas ${ }^{7}$ con masato y totuiropari o moka ${ }^{8}$; les cortaban el cabello, como un cambio y en señal de que ya no se es una niña, sino una mujer. También empleaban otras plantas como el ojé o potogo para aumentar de peso.

Con la primera conformación de los centros educativos, las jóvenes (sobre todo las que han ido y van a estudiar en los internados o a las ciudades) ya no aprenden a hilar algodón. Actualmente, hay pocas mujeres jóvenes que aprenden a hilar porque la reclusión se da pocos días, sólo lo que dura la menstruación, se prioriza lo que se aprende en los centros educativos.

Como se dijo inicialmente, antiguamente las prohibiciones no sólo se daban en la primera menstruación, sino en todas. Tradicionalmente cuando una mujer reglaba se quedaba un poco aparte andando por su propia trocha, entraba a la casa por otra puerta y se sentaba en un rincón para evitar contaminar el

6 El huito es un fruto comestible que también es empleada la infusión de la parte carnosa rallada para pintarse el cuerpo de negro. Lo curioso es que al echarte el líquido a la piel no se tińe directamente, tiene que pasar al menos unas 4 horas para que se ponga negra la piel. Esta pintura puede durar tres semanas.

7 Se ha encontrado una referencia sobre el uso de una bebida llamada Tsigoroki, arbusto del cual se utiliza su raíz. Lo rayaban y chapeaban (cernido) con un trapo para extraer el jugo. Luego se vertía en un envase que ya contenía masato. A partir de las dos de la mañana se le exigían a las recién menstruantes beber hasta quedar vacío el depósito con el propósito de tener mejor salud (Proceso de desarrollo de la Comunidad de Camisea, s.f). También kitsókiri, una especie de plátano grueso. Se siembra mayormente para dárselas a las mujeres cuando llegan a la pubertad y hacerlas vomitar con el fin de que sean gordas.

8 Se toma rallado con el masato dulce. Lo emplean para engordar y tener una vida saludable. suelo. Cuando los hombres veían o pisaban la sangre de menstruación o del parto, o tenían relaciones sexuales, ellos se volvían débiles y como enfermos con reumatismo. Perdía su energía viril y con ella perdía la puntería (Instituto Lingüístico de Verano [ILV], 2011: 505). Para evitar estos síntomas se bañaban con iraatsipirini, de esta forma la sangre que estaba en el cuerpo del hombre se desvanece. En el caso de las mujeres, no se conoce que sean vulnerables a la sangre menstrual de otras mujeres (Izquierdo, 2001: 126).

Otro punto importante que fue comentado en relación a la dieta, es que cuando las niñas ya empiezan a mostrar indicios de la formación de sus senos y caderas tiene que empezar a dietar para que pueda desarrollarse bien y no empezar a menstruar a una edad temprana. Anteriormente se creía que al tener la menstruación significaba que ya se había iniciado a tener relaciones sexuales. Es así que desde que empiezan a reglar ya pueden juntarse con un hombre, es decir ya pueden tener un marido.

Un tema poco comentado sobre la menstruación fue la prohibición de acciones de trabajo de la mujer, referidas a la agricultura. Las mujeres menstruantes no pueden recolectar los frutos de los árboles, se dice que esto provocaría que los frutos se echen a perder, el árbol empezaría a dar malos frutos partidos. Al igual que no se puede ir a la chacra y trabajar los cultivos, sino la chacra quedaría sucia, se morirían las plantas que recién fueron cultivadas. Esta información sugiere que las mujeres tienen que guardar cierta complementariedad en sus ciclos reproductivos con los de las plantas. Una mujer que está menstruando está en un proceso de limpieza en que, por la sangre, es expuesta y visible a los espíritus del bosque. Por ello la menstruación es un periodo para el reposo y posteriormente entrar a la fecundidad.

\section{Prácticas y costumbres durante el embarazo}

Existen prohibiciones que han sido nombradas por la gran mayoría de mujeres y hombres de Camisea, las cuales también han sido escuchadas en otras comunidades Matsigenkas del Bajo Urubamba. Sin embargo, son las prohibiciones relacionadas con animales pequeńos, los frutos que fueron expresados en matsigenka y las relacionadas con la luna, las que fueron compartidas por parteras, ayudantes de parteras, hombres mayores; información descrita también en 


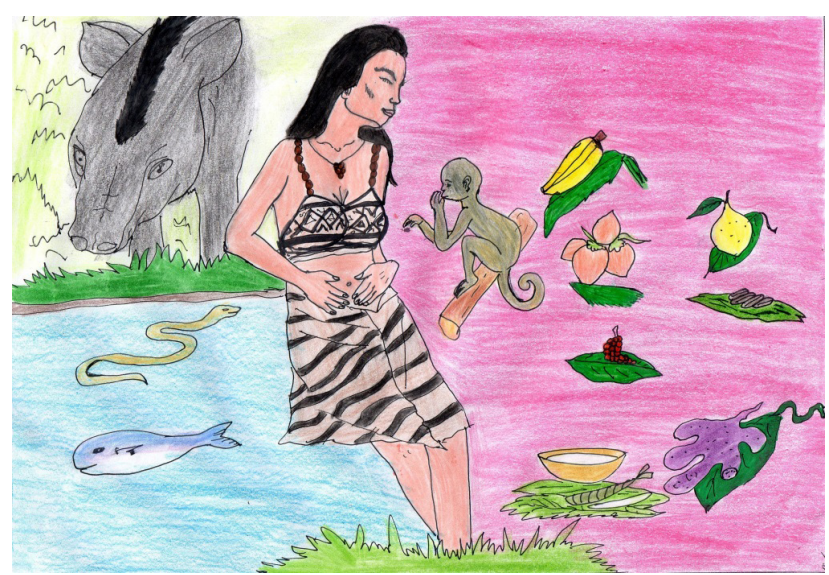

Dibujo 1. Lo que no pueden comer las mujeres. Yaquemilsa Matiashi Vicente, 2017.

el diccionario Matsigenka-castellano del Instituto Lingüístico de Verano (2011) y la investigación de Izquierdo (2001).

Los Matsigenka creen que el destino de los niños puede estar determinado mientras están en el vientre de la madre (Izquierdo, 2001: 118). Durante los tres primeros meses de embarazo la mujer debe de evitar de consumir el plátano guineo porque el bebito puede engordar mucho y no pueda salir al momento de dar a luz; la carne de tapir, majaz, y sachavaca (y ahora también se suma a la restricción, no comer carne de ganado vacuno), los frutos coco, castańa, poroto (en el caso del frijol porque aumentará el líquido de su cabeza) dado que pueden provocar el crecimiento de la cabeza o cuerpo; venado y rana (tonsanto), al bebe le ocasiona que nunca le salgan los dientes (Baer, 1994); monos, tucanes y otros animales de color negro porque saldrá moreno'; comer pan de árbol, el niño puede nacer todo lleno de grasa blanca en las axilas; evitar de comer chancaca, harina de yuca, arroz quemado (ILV, 2011: 523) para prevenir que la placenta no se pegue en el útero. Los peces que tienen dientes, están prohibidos para las mujeres embarazadas como el sábalo (mamori), paco, bazaco, andanchoeta, creen que pueden morder el estómago y causar diarrea o sangre en las heces (Izquierdo, 2001:184). Asimismo no pueden comer soremanteki (parecido a la granadilla) y maracuyá porque el niño será encerrado en la bolsa fetal como esa fruta cubre a sus semillas con el mesocarpio.

Larvas como las especies koshanti y tsuro chagárento, están prohibidas comerlas para evitar que nazcan hijos

9 La planta küitaeguiri fue nombrada por una madre de Camisea, la cual es empleada durante el embarazo para que el niño sea «blanconcito». «sin cuello», o con cuello muy corto y los hombros encorvados cerca de la cabeza, y también para evitar que salgan cabezones lo que dificultaría o impediría el parto (ILV, 2011: 235) y una posterior hemorragia durante el parto.

No se come el fruto caimito, al ser pegajoso cuando sale el niño la madre sufre, y se demora. Tampoco tsigaro (shapaja) porque no podrá salir rápido, ni manataroki (shika shika) porque puede quedarse entrelazado con su cordón umbilical. En cuanto a otros vegetales en Baer (1994), está registrado que si se come la sachapapa roja, el niño no podrá hablar, o a lo sumo tartamudeará.

Aunque antiguamente no había azúcar, el consumo de sal y dulces también era prohibido en el embarazo y postparto (hasta que cayera el cordón umbilical), incluso algunas mujeres comentan hasta tres meses después. Al considerar los dientes débiles en ese periodo. El hombre también era implicado en no comer sal cuando su mujer daba a luz, pero no tanto tiempo como la parturienta. Actualmente esto sólo se da durante la primera menstruación.

Los varones no podían comer frutos que son gemelos, dos en un mismo lugar, porque cuando tengan hijos nacerían gemelos, también estaban prohibidos de comer tsimeri hembra (pájaro pequeño). Las prohibiciones de los padres están más relacionadas con algunas actividades que con la alimentación, como el caso de hacer o poner brea a sus canoas y flechas intsinetara (Izquierdo, 2001: 120) porque esto podría provocar que la placenta se quede pegada en el vientre; tampoco deben cazar tigre porque luego no podría caminar la criatura; ni matar serpientes porque la criatura nacería inválida o sin fuerza. Asimismo el padre no puede tocar la madera de catahua (kamana), la fuerte resina puede quemar la piel del bebé. El bebé puede caer enfermo y su cuerpo llenarse de ampollas (Izquierdo, 2001: 119).

Una restricción común para la madre y padre es el no tocar la liana llamada tapetsa (tamishi), con la que se fabrica cestas. Se dice que este material pajizo hace sobresalir el ombligo de la madre (Izquierdo, 2001:120). Así como este tipo de prohibiciones habían muchas otras en torno a la realización de ciertas actividades, el padre no podría desafiar ninguna de ellas porque ponía en riesgo las vidas de la madre e hijo. Los padres antes de que naciera el bebé y hasta que se le cayera el cordón umbilical, debían de trabajar duro, realizar actividades de fuerza como amontonar 
la leña, para que cuando nazca el niño no sea ocioso (Baer, 1994) ${ }^{10}$.

Existen algunas prohibiciones relacionadas con la luna, si esta es mirada en luna llena, cuando aparecía grande en el horizonte, el feto podría crecer tan grande que no podría nacer. También la estrella Porinti, que según la mitología Matsigenka es conocido como el último hijo de Luna y hermano menor del sol; se decía que el marido de una mujer embarazada no debía seńalarlo con la mano, porque de lo contrario, podría hacer abortar o tener un mal parto a su esposa, y así llevarse a su bebé (ILV, 2011: 397).

Mantener las reglas de comportamiento y las restricciones alimenticias aún son creídas como medio de prevención para el desarrollo del feto ${ }^{11}$ pero también para evitar rasgos negativos del comportamiento de los nińos, como son la ira y la pereza (Izquierdo, 2001: 119). Igualmente como menciona Baer (1994), si el alma del bebé es capturada y llevada por un nohumano, el bebé nacería muerto. Si el bebé no pierde su alma, la influencia no-humana produce cambios físicos y/o discapacidad mental. Tener un labio leporino es un tipo de evidencia física característica considerada de la influencia de un demonio, kamagari, la formación de los recién nacidos. Se dice que antes los bebés, que nacían con deformidades, eran asesinados porque podían llegar a ser peligrosos, ya que no sólo afecta el físico, sino también la personalidad del recién nacido, que sería en parte humano, y parte demonio. Las influencias de no-humanos, sin embargo, no necesariamente producen cambios físicos, también pueden producir cambios mentales que sólo puede hacerse evidente durante el desarrollo infantil que afectan a la forma y expresiones.

Otras prohibiciones generales que son actualmente promovidas por los promotores y el personal de salud son: no tomar masato fuerte, no jugar pelota, no tomar medicamentos, antibióticos o plantas medicinales (sangre de grado, uña de gato, tiroti, kantiare y otras), bañarse a diario, no usar ropa apretada, durante el

10 La partera de Camisea hizo referencia de acciones diferenciadas según el género del feto y/o recién nacido. Los padres tienen que trabajar más cuando es mujer la bebe porque al hombre le da pereza y quiere dormir, por esta razón el hombre tiene que ser fuerte y trabajar duro. Igualmente en el caso de las madres les da pereza cuando el hijo es varón, y ella a la fuerza tiene que hacer algo porque si no lo hace será más perezosa.

11 Yatsatake significa estar o ser afectado por lo que su madre había comido o hecho durante el embarazo. Yatsati ananeki tatarika ogatetakari iriniro, un bebé adopta las características de cualquier cosa que su madre haya comido durante el embarazo (ILV, 2011: 523). octavo y noveno mes ya no tener relaciones sexuales. Las madres recomiendan consumir durante el embarazo el masato dulce de forma continua. Alimentos para llevar bien un embarazo son chapo pescado, chopispa, caldo de carachama, o caldo de toteroki (caracol) el cual es mencionado también para prevenir la anemia y cuando se tiene alguna infección. Baer (1994), igualmente menciona que es permitido comer corazones de algunas palmeras, como kamona (Iriartea sp.) y tiroti (Astrocaryum sp. $)^{12}$, y pescados de pequeño tamaño, sin dientes (bagre, chiripira).

\section{Prácticas y costumbres durante el parto}

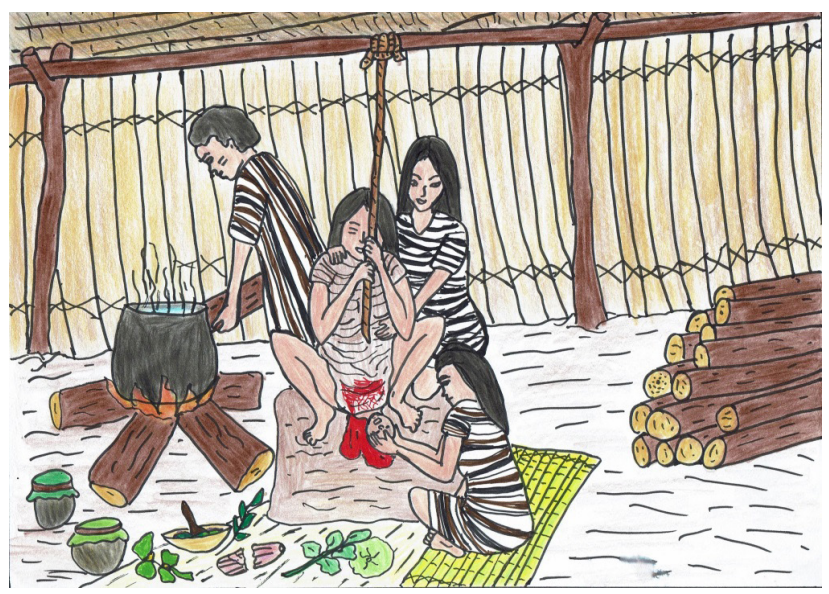

Dibujo 2. El parto antes. Yaquemilsa Matiashi Vicente, 2017.

El parto es un evento que se da en familia. Aunque actualmente la mayoría de los partos son atendidos en el centro de salud, esto no deja de lado la participación de algún familiar acompańando a las mujeres embarazadas. No obstante, dar a luz a solas, y sin gritar, es reconocido como fortaleza y expresión de seguridad entre las mujeres. En la menarquía se les decía que no tenían que gritar en su primer parto porque sino los siguientes serían dolorosos. Las mujeres reconocen el momento de pujar cuando ya no se aguanta más dolor en la cintura, la barriga se pone dura y la cabeza está muy caliente. Otras también lo relacionan con las ganas de defecar.

No hace menos de tres décadas que aún las mujeres de Camisea daban a luz en el bosque. Las mujeres se

12 Alimentos que tienen que ser buscados en el bosque, alejados de sus residencias. Por eso actualmente son pocas las mujeres y hombres que los nombran porque implica una actividad física que los hombres ya no realizan durante el embarazo y puerperio de sus parejas. 
agarraban de las ramas de árboles, no muy altos como el cacao, y haciendo fuerza lograban dar a luz. Se ponían a cargar cosas pesadas como el comegen (nido de termitas) o troncos, el mismo día que empezaban con los dolores de parto. Luego regresaban a sus casas con su bebé. Pocas veces se avisaba a los hijos, en algunas ocasiones eran acompañadas por la hija mayor o la hermana para pedir apoyo en el corte del cordón umbilical de alguna persona de la comunidad.

Antes era un acontecimiento netamente femenino, madre, tía o abuela y partera apoyando en el primer parto. Aunque no es impedida la participación de los hombres, antes estaban prohibidos de recibir a los bebés porque no debían de ver la vagina o tocar la sangre del parto o el sangrado de la mujer. Podían ayudar trayendo la soga o el palo para que la mujer se sostuviera o estar por detrás, sosteniendo a la mujer por la espalda, pero nunca estando delante de la mujer porque esto podría originar que luego no pueda cazar, ni traer pescado, en consecuencia no traer carne a la familia. Los animales se le escaparían o se esquivarían porque podrían olerlo.

Antiguamente culminado el parto se cortaban el cabello, señal que el dar a luz es un cambio en la vida, y se hacían purgar para limpiar el interior del cuerpo. Luego de una semana, cuando cae el ombligo del bebé, la mujer ya podía empezar a trabajar para que no fuera ociosa. Aunque desde el primer día luego del parto las mujeres comienzan a hilar algodón con el mismo fin.

A partir de que se inició la atención en el puesto de salud, los partos cambiaron de lugar. Son cerca de treinta ańos de atención en Camisea, las abuelas recuerdan cómo médicos y enfermeras las atendieron a algunas de ellas amarrándolas y tocándolas mucho previo al parto.

Actualmente (2016), basándonos en la información estadística del sistema de información salud de la Red de Servicios de la Convención, se puede conocer que el porcentaje de partos institucionales en el 2015 de la microred de Camisea fue el 66.5 \% (249 de 374 partos). Asimismo el porcentaje de partos institucionales en el primer semestre del 2016 fue de 62.5\% (117 de 187 partos).

Como se señala un estudio de línea base en salud nutricional de los nińos de la organización Ayni Desarrollo (2016: 12), realizado en las comunidades de Timpía, Miaría y Puerto Huallana, se identificó que el $91.9 \%$ de los partos se atendió en el establecimiento de salud. Es muy importante seńalar que sólo el 13.6\% de estos partos se produjeron en posición vertical. Así que se puede evidenciar que no todo el personal de salud de la microred está preparado para la atención del parto en posición vertical.

Mujeres mayores y jóvenes, entre los 14 y 42 años de edad, van a dar a luz en el centro de salud. Con la información estadística proporcionada por el personal de salud, se reconoce que casi la totalidad de los partos en la comunidad son institucionales. Las mujeres han decidido parir en el centro de salud. Existen distintos motivos por los que se ha optado no parir ya en las casas: para prevenir complicaciones, por tener gastritis, al ser un embarazo adolescente, un parto prematuro, tener preclampsia, por pasar los cuarenta ańos de edad, porque hay muchas personas en la casa, entre otras.

En testimonios de mujeres de Camisea que dieron a luz en el puesto de salud, se consigue identificar que la mayoría da a luz en posición horizontal y en la camilla. En ocasiones les obligan a cambiar su ropa por una bata. Algunas han sido alarmadas durante sus embarazos por cómo estaba posicionado el feto en los últimos meses o por tener alguna complicación de la salud como la gastritis. Les han puesto suero antes de dar a luz, que posiblemente sea con oxitocina para apurar el parto. Se les ha practicado la episiotomía, cuando la atención ha sido fuera de la microred de Camisea. Sin embargo, ocasionalmente se da con la participación de la partera y la familia, pudiendo hacer el uso de plantas medicinales.

Conviene subrayar que el aumento de los partos institucionales es a través del discurso oficial del personal de salud sobre cómo deben ser y en dónde, con el propósito de prevenir las muertes maternas y perinatales. Se suma a esto la desvaloración del conocimiento local de la partería por las mujeres jóvenes, no sólo porque ya no hay una participación más activa de las parteras y promotores de salud, sino porque las adolescentes ya no aprenden durante su primera menstruación la totalidad de conocimientos que antiguamente se transmitía durante este periodo: cómo cuidarse como mujeres y como madres, qué plantas utilizar para su salud, cómo aprender a tener paciencia, la importancia de las purgas y dietas para el cuidado del cuerpo en periodos de transición. Igual perjuicio tuvo el cultivo y empleo de las plantas invenkikis, al ser prohibidos por las misioneros evangélicos y católicos dominicos. Actualmente estas plantas son conocidas por pocas personas y su conocimiento es reservado para la familia. 
Lo que además manifiestan las mujeres es la preocupación de tener una complicación previa al parto y por ello ser trasladadas a Lima para una cesárea. Por este motivo buscan otra opinión de la partera sobre el diagnóstico ofrecido en el establecimiento de salud. Aunque al mismo tiempo ya no existe la plena confianza de lo que la partera les dice. En los primeros ańos de conformación de la comunidad hasta la fundación del centro de Salud, los partos se realizaban en casa y a nivel familiar, las parteras asistían en los primeros partos o los que tenían alguna complicación. La labor actual de las parteras se ha reducido en acomodar a los bebes y estar presente en el parto, pero no teniendo una participación activa en la atención cuando este se realiza en el puesto de salud.

\section{Facilitar el parto}

El usar clara de huevo con malva es generalizado en las comunidades del Bajo Urubamba. Algunas mezclan el huevo (y de preferencia de gallina negra) y malva, o malva con sábila, hasta que ambas tengan una consistencia flemosa y es tomado cuando se inician las contracciones. Según las mujeres, al tener una consistencia resbalosa ayuda a que el bebé salga fácilmente y la malva ayuda a calmar el dolor.

Las hojas de malva también son utilizadas como mate, al igual que la corteza de la planta shinti (topa o yausa pancho), y con agua caliente se soba el vientre. La consistencia es flemosa. Otros mates empleados son la manzanilla, anís y coca los que alivian el dolor. También ishanguearse (frotarse) con ortiga sirve para aguantar el dolor cuando empiezan las primeras contracciones.

Otra planta comúnmente usada es el cacao rojo (kirajairi), su semilla es raspada y se toma en mate, otros emplean la corteza complementándolo con el piripiri, éste último se conoce en toda la selva peruana con el nombre en quechua piripiri, en matsigenka, se nombra ivenkiki $i^{13}$. Aunque no todas las mujeres comparten sus ivenkikis, las parteras los emplean, inclusive cuando el parto se lleva a cabo en el centro de salud.

13 Como lo han detallado las mujeres en las conversaciones, el ivenkiki es una planta que se cría, al igual que un hijo o un animal. Es por eso que muy pocas mujeres lo comparten. No necesariamente porque no quieran ayudar, sino porque la planta no va a tener el mismo efecto si no pertenece a la familia. Los ivenkikis hay de muchos tipos. Sólo el ojo de quienes los cuidan saben reconocer cuál es y para qué sirve. Se crían lejos de los caminos, de la basura o las gallinas. Por eso están en las chacras.
El ivenkiki se mastica o se tritura, se puede tomar con un poco de agua tibia o consumir sólo el jugo o también se puede emplear la mezcla de agua tibia con el jugo extraído para frotarlo en el vientre. Además de emplear plantas, se realizan masajes para poner en buena posición al bebé, el bañar con agua caliente a las parturientas para resistir los dolores de las contracciones. Es muy importante que no le entre frío a la mujer cuando ha iniciado su proceso de parto. Como acciones preventivas están todas las que se deben de realizar durante el embarazo.

Las primeras personas que conformaron la comunidad recuerdan cómo era antes de la presencia del personal de salud, cuando existían complicaciones en el parto se usaban plantas, que aún hoy se usan pero son menos conocidas por las madres jóvenes. Sin embargo el uso de las plantas es promovido por las obstetras que tienen más tiempo de experiencia trabajando en comunidades amazónicas. Como nuevos elementos considerados previos al parto están el caminar mucho, concentrarse en su respiración, algunas mujeres han experimentado comiendo chocolates, otras informan les han puesto inyecciones para el dolor y han tomado pastillas para bajar la presión.

Cada mujer decide si quiere hacer empleo de plantas medicinales. Las madres más jóvenes algunas veces dudan de su efectividad.

Algunos ya no creen y ya no usan piripiri pero cuando toman y ven cómo funciona. Algunos en su chacra tienen sus medicinales. Las jóvenes de 20 años, 15 años, menores de edad dependen de su madre para que tome y nazca rápido (A. Silvano, entrevista, 25-06-2016).

\section{La placenta y el corte del cordón umbilical (moguitontsi, ombligo)}

Cuando el bebé ha nacido se espera a que la placenta salga y el cordón umbilical deje de latir para que éste sea cortado. La partera de Camisea y, al igual que las de Timpía, explicaron que cuando ellas atienden los partos «ordeñan» el cordón haciendo que pase la sangre al nińo.

Si yo le he dicho sobar aquí para que no se va su sangre, para que se vaya a su cuerpo, ahí recién lo ha amarrado (Luzmila, entrevista, 04-03-2016). 
El corte del cordón umbilical es al momento, cuando sale el bebito le ponen el clamp y rápido hasta antes de que salga la placenta. Pero los machiguengas esperamos hasta que salga la placenta y recién le cortamos el ombligo. Pero los que atienden en el establecimiento el corté es rápido, recién al salir el bebito. Eso no se debería hacer. Yo cuando no está el doctor, igualito espero que salga la placenta para cortar el cordón, cuando está el doctor yo hago lo que él hace. Yo preferiría cortar cuando salga la placenta (R. Semperi, entrevista, 26-10-2016).

El corte del cordón umbilical antes era realizado por la partera, abuela, hermana, padre o un familiar cercano. Se hacía con el pedazo de una paca o caña bien filuda o estrangulando el cordón con el hilo de algodón (manvetsa). El ombligo era amarrado con el algodón y con la medida de dos dedos se determina dónde cortar.

Actualmente se estila pedir a un familiar o conocido para que realice el corte y este sea la madrina o padrino del bebé. Algunas de las obstetras apoyan esta práctica siendo ellas las madrinas o esperando a que se acerque la persona que ha sido elegida por los padres para que ésta realice el corte.

Luego se destina a enterrar placenta, se guarda en una bolsa, o envuelta en hojas de plátano y se entierra en un hueco profundo con piedras para que sea imposible que los animales puedan comerlo.

Anteriormente, se creía que la placenta se enterraba lejos para que el bebé no la escuchara ${ }^{14}$. Se dice que si eran tiradas al río podrían comérsela los peces y eso podría ser la razón por la cual el niño se enfermaría, a su ombligo le saldría heridas y se infectaría, o podría provocar que la mujer tuviera muchos hijos. Algunas mujeres han botado al río su placenta, pero la partera y las mujeres mayores explican que es importante para la salud de la madre y el recién nacido que la placenta esté bien enterrada.

Fueron nombrados varios lugares dónde enterrar la placenta: cerca de la casa, en la parte arenosa cerca del río, en el bosque a los pies de un árbol o planta de crecimiento rápido o fuerte como el pijuayo o tairi. Quien se encargaba era una de las primeras parteras, ella las enterraba en donde queda actualmente la garita de la comunidad. Ese espacio estaba lleno de árboles y enterraban las placentas cerca de una planta de plátano.

14 No se ha podido encontrar referencias si la placenta actuara como un alma de un humano muerto que está en busca de compañía para viajar al mundo de los muertos.

\section{Dificultades del parto, formas de solucionarlo}

La espera de la placenta a veces toma su tiempo, es una de las preocupaciones principales frente a una posible hemorragia. Es quizás por esta razón que existe una gran cantidad de maneras de hacer que la placenta salga prontamente y remedios para reducir las hemorragias postparto.

Si la placenta demora en salir, se la induce a que salga dando golpes en la parte baja de la cintura con una escoba de palmera o cinturón de cuero; sobando con el calzoncillo del padre (no necesariamente el que esté usando) o con un pescado similar a una anguila, también se da de lactar, pero los que nombran más efectivos es soplar fuertemente en el pico de una botella y tomar ceniza (samampo) cernida y mezclada con agua tibia y limón.

Igualmente existen muchas plantas medicinales que permiten que salga la placenta:

Yaviro, especie similar a la pińa, primero se tiene que machucar las hojas y luego hacerlo hervir y tomar una tasa de la preparación. Es una planta que crece en las partes altas por eso no está en Camisea, la consiguen en sus viajes al Alto Urubamba. Se la toma durante un parto cuando no se cae rápidamente la placenta; al tomarla, debe caerse inmediatamente; algunos la conocen por el término notaretsiriantite pińa para la placenta (ILV, 2011: 596).

Shirimogútoshi (Santa María) tiene varios usos, para el baño de la madre después del parto y también es usado como el yaviro, ambos funcionan muy bien. Se toma como mate, se tiene que hacer hervir las hojas, sirve para cicatrizar adentro de la vagina y no estar hinchada. Lo particular de esta planta es su nombre, se llama literalmente planta ombligo, Moguto o Shirimogútoshi (hoja para caer el ombligo) es una hierba conocida también en el Bajo Urubamba con el término inarópini por la forma de las hojas. Las partes de la hoja son: omogutotsa su pedúnculo (su cordón umbilical); omoguto su ombligo (donde se unen oshitsa las venas destacadas de la hoja); iranotare ananeki la forma de la hoja (la placenta de un niño). Algunos usan el nombre tsivetápini (planta de raya) para referirse a esta planta. Tiene hojas grandes con la forma de un pez raya o una placenta; también tienen una concavidad pequeña donde se conecta con el pedúnculo que parece un ombligo. Se prepara mate de las hojas que las mujeres toman cuando tienen dolores de parto, y la placenta no quiere bajar; también se toma para bajar 
la hinchazón postnatal del útero. También se utilizan las hojas para preparar baños calientes para calmar los dolores postnatales y remojar los pies para aliviar el reumatismo, resfríos y la picadura de raya (ILV, 2011: 459).

Potogo (ojé), del mismo modo, sirve para bajar la placenta. La resina se hace hervir primero se toma una cucharadita.

La raíz de la Sara-sara (sarioki) y el Pocharoki, se cocinan para tomarlo en mate con el mismo fin.

Otras plantas empleadas luego del parto para prevenir las infecciones y hemorragias son:

Omogútotsa inkite, esta planta es un bejuco que se tiene que triturar y luego hervir para ser empleado para bañar a la mujer parturienta. Este bejuco tiene mellas que parece son útiles para apoyar el pie y que le da la apariencia de una escalera. Su nombre significa el cordón umbilical del cielo, esta planta es nombrada en los mitos Matsigenka sobre la relación de seres del cielo con los matsigenkas, habitantes de la tierra. Tradicionalmente usado para prevenir la infección después de un parto. Por su apariencia, antiguamente se decía que este bejuco era parecido a una especie de cordón que mucho tiempo antes había conectado la tierra con el cielo. Este cordón tenía unos escalones como de una escalera, por medio de la cual los que vivían aquí en la tierra habían podido hacer visitas a los inmortales que vivían en el cielo. Según se contaba, el cordón fue cortado por los inmortales cuando fueron engañados por el mentiroso caracol Machoeri y desde aquel entonces, no solamente se había ido aumentando la distancia entre la tierra y el cielo, sino que ya no se sabía cómo llegar a Meshiareni, la fuente de la vida eterna (río de cambiar la piel). El afán de muchos matsigenkas, especialmente de los chamanes, al través del tiempo, era encontrar otra ruta para llegar allá (ILV, 2011: 326).

Kosamati (sangre de grado) se toma su resina y se utiliza baños de asiento, también se cocina la corteza y luego se toma; Pakitsápari ${ }^{15}$, es una planta que se encuentra en los montes, difícil de encontrar, se utiliza su raíz, tiene que ser cocinada y después se toma, ambas reducen la hemorragia.

15 Este arbusto también se decía que era utilizado para ayudarle a uno tener buena puntería y a los perros a ser buenos cazadores. Se la raspa, se mezclan las virutas con agua y se toma la medida correspondiente; después se toma el masato fresco para provocar el vómito. Se emplea el término vigaatagantsi para referirse a la acción de tomarla porque es muy tóxica (ILV, 2011: 349).
También se toma la planta Iraatsipiri con el mismo fin, se toma la cocción de la hoja. Esta planta también sirve para que baje la menstruación, cuando se quiere tener hijos otra vez.

Iraatsivenkiki, se utiliza para parar la hemorragia, se tiene que machucar y con agua tibia se toma.

Samponerotsapini, sirve para la hemorragia cuando se ha dado a luz, ayuda a reducir la hinchazón del vientre. También se usa cuando hay sangrados por un aborto y para la menstruación abundante.

\section{Dietas y cuidados después del parto}

Al presente no todas las mujeres realizan la dieta alimenticia y los cuidados después del parto. Antes las parturientas se cuidaban cerca de un mes con la ayuda de sus madres, quienes las apoyaban en los quehaceres de la casa y la preparación de los alimentos. Ahora hay algunas mujeres que se cuidan sólo tres días o una semana, esperando la caída del cordón umbilical.

La alimentación principal de las madres es a base de líquidos como los caldos de gallina, pescado, toturoki (caracol); masato y chapo frescos los que pueden promover la generación de leche. Durante la lactancia también se prohíbe comer dulces, alimentos con sal (se le ha agregado a esta dieta las frituras), al menos durante el primer mes, para evitar la caída de los dientes. Tampoco se debe comer animales que contengan dientes porque dicen que se aumentará la sangre y la mujer tendrá niños seguidos. Si se come caña de azúcar, al bebé le puede dar alergia y salirle granitos en el cuerpo; comer la oncucha y piña le sale aftas $\mathrm{u}$ hongos en la boca y lengua; papaya porque le puede producir diarrea; si come sachapapa roja el niño no va a poder hablar.

La planta que más se emplea para aumentar la producción de leche, en las comunidades Matsigenka del Bajo Urubamba, es puigoro. Se lavan los senos con agua tibia y también toman la infusión por tres días. Igualmente toman el mate de la raíz de pijuayo para aumentar la cantidad de leche. Este mate también sirve para sanar las heridas de los pezones.

\section{Cuidados del recién nacido}

Existe un variado número de plantas que se emplean para la salud, crecimiento y cuidado de los nińos. 
La ceniza de okago shinki (coronta de maíz), se emplea para que el ombligo cicatrice rápido; kosetsipini para los niños que tienen afta, se tiene que raspar su tronco, ser cocinado o empacado y luego usar un poco de algodón, también se puede usar ceniza y con un pedazo de algodón humedecido quitar las aftas; yanaivenkiki para que camine rápido antes de que amanezca se le bańa en agua tibia; kamparoivenkiki para que pueda dormir, se le baña con el agua tibia en la tarde; shiarontsivenkiki para la diarrea, se toma con agua tibia; pocharovenkiki para que el niño engorde; maviki, para caminar en la noche con el bebé y no enfermarse; flor de choropetatava para bañar en la primera semana para que no enfermen los bebés, pueda caminar, se pueda llevar al bebé; kitetegari, es una planta de flores amarillas de olor fuerte que se emplea para bañar a los bebés luego de haber escuchado que alguien ha fallecido o si lo has llevado a tu bebé donde está un fallecido. Esto se emplea para prevenir que los espíritus malos puedan asustar a los bebés ${ }^{16}$.

Una de las mujeres de Camisea comentó que antes para que los bebés crecieran sanos no podían salir de la casa al menos tres meses, limitando que estos fueran vistos por otras personas que no sean los parientes cercanos. Ni ver la luna llena porque según las historias, la luna se lo puede llevar y comérselo ${ }^{17}$.

El estar atentas a los bebés es parte de la cuidados de los recién nacidos pero sin llegar a sobre protegerlos porque no sería bueno para la formación del niño.

... lo amamanté. Luego lo dejé que se descanse bien abrigado para que no le entre frío. Siempre es la costumbre que no le tenga el niño recién nacido en tus brazos o en tus rodillas porque se dice que cuando crezca no será trabajador y será haragán... Debes dejarlo por unos momentos luego de lactarle, lo dejas que este se descanse hasta que llore (Shina, entrevista, 06-12-2014).

16 Otro hecho importante sobre el cuidado del cuerpo de los recién nacidos en comunidades del pueblo Matsigenka de la cuenca del Manu, Madre de Dios, como lo detalla Shepard (2002: 207), para los Matsigenka los penes grandes no son un signo de virilidad, sino la manifestación de la sexualidad agresiva, los demonios en la mitología Matsigenka se describen poseyendo penes enormes. Las mujeres Matsigenka a veces bañan a sus hijos recién nacidos con una infusión de especies de orquídeas que tienen pequeñas bombillas para que crezca hasta tener un pene de tamaño normal, es decir, pequeño. Si uno fuera a emplear orquídeas con grandes bulbos fálicos, el niño podría crecer hasta tener un pene grande y un apetito sexual demoníaco.

17 Esto hace referencia al mito de Luna cuando se volvió caníbal al ser obligado por su suegra comer a su esposa muerta luego de dar a luz dos gemelos. Por eso se considera que la luna puede estar merodeando por asentamientos Matsigenka para poder coger un humano desprevenido o en un periodo donde exista cierta vulnerabilidad.
En ocasiones se observan en la comunidad de Camisea a los niños que recién empiezan a caminar que tienen amarrados en su cuello su ombligo o un ivenkiki a manera de protección. Existen varios insectos que son considerados peligrosos para la salud de los niños. Usan la planta petaguirishi en los baños a los bebés para que no sean cutipados por hormigas ${ }^{18}$, yairiivenkiki cuando es por las abejas yairi, así evitan las diarreas, fiebres o vómitos de los nińos. No solamente se previenen de estos animales sino también de algunos fenómenos naturales como la niebla, amokari, utilizan ivenkikis como amuletos.

Son varias hormigas que cuando el bebito se pone mal... el primero es sankori, maseri, después kaveti y petyagiri, son cuatro hormigas que lo cutipan, que dañan a los nińos, a veces ahora te das cuenta que todos los niños están con su amuleto con piripiri para contra de esos animales y para cuando empieza a nublarse para que no le cutipe también el nublado amokari... también tiene su secreto, el piripiri se llama amokarivenkiki,... pero yo casi no he utilizado en mis hijas, por eso yo lo que les veo les pregunto me dicen que esto, sirve para esto. También utilizan piripiri para yairi, yairivenkiki eso le echan al bebito, el yairi es un mosquito. También le puede llevar dónde está ese nido el bebito y empieza a llorar llorar y se puede morir por el llanto como según dice costumbre que le pica, es como animal malvado es un ave ${ }^{19}$ malvado... su alma al bebito se lo lleva, cuando

18 Se piensa que las hormigas (petyagiri, sankori, manii, yamposhito, kaveti, tsivokiro), enferman a los niños llevando migajas de sus comidas o las deposiciones o caminando por sus heces u orina. De éstas, las petyagiri se consideraban ser las más peligrosas porque, al igual que las abejitas yairi, hacen sus nidos arriba en los árboles desde donde se decía miraban a los niños que pasaban por abajo, Aunque éstos estuvieran cargados por sus madres, y les quitaban sus almas haciéndoles enfermarse hasta morir (ILV, 2011: 71). La hormiga petyagiri es una hormiga negra que camina para adelante y atrás. Sankori es la hormiga más peligrosa que ocasiona diarrea con sangre.

19 Cuando hizo referencia de un ave quiso referirse a Tsiroti uno de los más grandes picaflores que habita en los cerros. Se decía que este picaflor era el yerno de la abeja yairi y que siempre se jactaba de él diciendo que su suegro podía hacer daño con sus flechas porque era capaz de hacer cualquier cosa; también se decía que cuando se veía al picaflor pasando a cada rato, ya se sabía que por allí estaba su suegro, y por lo tanto había que cuidar bien a los niños, y aun las personas adultas corrían riesgo de ser flechadas por yairi o por tsiroenti que aprovechaba que su pico era puntiagudo para utilizarlo como flecha. Si tsiroenti flechaba a un bebé, éste comenzaba a sentirse mal del costado; el remedio que se usaba era conseguir rizomas de piripiri tsiroentivenkiki, se los machucaba, se los mezclaba en agua caliente y se utilizaba esto para bañarle. También se decía que a veces el alma de un nińo andaba vagando y si se encontraba con tsiroenti, él se lo capturaba y lo llevaba a su suegro, yairi. Si una de ellas miraba a un bebé, aunque sea de lejos, podía picarle con su flecha y hacer que se enfermara y se muriera, mayormente de neumonía; para expresar esto se decía yagakeri (le ha llevado). También se pensaba que en caso de que la abeja yairi se llevara residuos de la comida de un niño, 
llora se lo haya ahí, tiene que el papá ir a tumbar ese árbol, botar a ese animal y el bebito se sana, porque empieza a vomitar amarillo amarillo, caga amarillo ${ }^{20}$ se puede morir la criatura». (R. Semperi, entrevista, 26-10-2016).

Izquierdo en su investigación (2001: 184) identificó que los Matsigenka consideran que algunos alimentos comestibles no son alimenticios debido a la creencia de que algunos animales son peligrosos. Estas creencias demuestran que incluso las enfermedades comunes tienen algunos elementos que se ajustan a la cosmología Matsigenka con respecto a la vulnerabilidad corporal y espiritual.

Es así como los problemas gastrointestinales se pueden asociar con un espíritu o la venganza del animal. Estas enfermedades no sólo afligen a la persona responsable, sino que los padres siguen restricciones en la alimentación para evitar contratiempos con sus bebés, al igual que la mujer embarazada evita ciertos alimentos para asegurar un parto exitoso. A los niños de una edad muy temprana se les enseña qué animales y plantas deben respetar, ya que comer el animal no es la única manera en que un animal puede vengarse. Burlarse de ciertos animales también resultará en daño al transgresor (por ejemplo moskini o el pájaro marini), se les dice que a estos animales no hay que imitar sus sonidos y les advertía que no se burlaran de ellos, sino ignorarlos (Izquierdo, 2001: 184) porque se pueden convertir en matsontsori (jaguar) y llevárselos.

\section{Reflexiones finales}

Conocer sobre las costumbres y prácticas relacionadas al parto, nos permite identificar que no sólo están sujetas a dicho momento, sino que están vinculadas con todo el ciclo reproductivo de las mujeres, el vínculo social y del entorno. Desde la menarquía se aprende a cuidar el cuerpo y emplear vegetales que favorecen la salud, la fertilidad y un parto con menos complicaciones. Se

sus heces o su orina, también cogería su alma la que llevaba a su nido y el nińo se enfermaba. El remedio era que el padre del niño tumbara el árbol donde pensaba que se ubicaba el nido de estas abejas. Después de tumbarlo, deshacía el nido con mucho cuidado para no hacer dańo al alma de su hijo ni hacerla huir, y al final quemaba el nido (ILV, 2011: 590).

20 Se atribuía la diarrea amarilla de los bebés a la abeja yairi diciendo que cuando cogía el alma del niño, le daba su chicha de maíz; también se pensaba que el espíritu protector de estas abejas (inato yairi) podía picar a los nińos y causar la neumonía. (ILV, 2011: 263). ha podido identificar las innumerables restricciones alimenticias y de acciones que tanto gestantes como parejas deben realizar para no afectar en la salud de los fetos y en cómo se da el proceso de parto. Dichas restricciones están vinculadas con la mitología Matsigenka, específicamente las que hablan de Kashiri (Luna), su mujer Matsigenka (la muerte de ella) y el nacimiento de sus hijos.

Las mujeres tienen una especial preocupación por las complicaciones del parto con hemorragias lo cual es destacable en el número de plantas mencionadas. Se ha podido reconocer también algunas plantas, que según su raíz lingüística hacen alusión a la placenta y al cordón umbilical, de esta manera se puede dilucidar no solo su importancia medicinal sino también cultural. La planta shirimogútoshi (hoja para caer el ombligo) es la más destacable y empleada luego del parto. Otra de las plantas es omogútotsa inkite (cordón del cielo), aunque poco mencionada por las mujeres de Camisea, es importante en la cosmología Matsigenka, al hacer referencia a la relación de los humanos Matsigenka con los seres del cielo.

En relación al lugar del parto, las mujeres Matsigenka de la comunidad de Camisea dan a luz mayoritariamente en el establecimiento de salud, el cual es atendido por obstetras mujeres. Como se ha señalado, las mujeres están de acuerdo, sobre todo las jóvenes, parir en el centro de salud, pero expresan su incomodidad en la atención porque no pueden parir en cuclillas y con su ropa; algunos de sus partos son apurados al igual que el corte del cordón umbilical; no siempre se permite la participación de la familia y las parteras; también por la manera de informar los diagnósticos de embarazos con riesgo sin el apoyo de una intérprete.

Simultáneamente a la atención recibida en el establecimiento de salud, las mujeres embarazadas solicitan el diagnóstico de las parteras para un posible acomodo de los bebés, sin existir una coordinación con el personal de salud. En comparación a otras comunidades donde parteras y promotores de salud participan durante la atención del parto, coordinando con el personal de salud, en Camisea se evidencia un desinterés por articular con las parteras y promotores, quienes podrían favorecer en comunicar a las gestantes los cuidados durante el embarazo, dar un mejor diagnóstico durante el embarazo y llevar a cabo bien las referencias de emergencias obstétricas al actuar de intérpretes. 
Por otro lado, hay que destacar que en la atención de las obstetras existe consideración con las mujeres para que empleen sus plantas medicinales para facilitar el parto, la posibilidad que dan a la familia para realizar el corte del cordón umbilical para el padrinazgo y la facilidad que dan a la parturienta y acompañantes para llevar a cabo el bańo-limpieza después del parto. Además, es reconocida la importancia de la casa materna para las mujeres gestantes de comunidades alejadas para que los embarazos riesgosos sean atendidos en el centro de salud y posteriormente dar seguimiento a las madres.

Estos avances, aunque limitados, corresponden con la aplicación de la actualizada «Norma Técnica de salud para la atención del parto vertical en el marco de los derechos humanos con pertinencia intercultural». No obstante, el personal de salud debería de informarse más sobre el empleo de los ivenkikis y otras plantas medicinales que favorecen el cuidado de las mujeres en edad reproductiva y la del recién nacido.

Del mismo modo se pone en evidencia que el sistema médico occidental sólo considera las plantas como un recurso biológico y ciertas prácticas culturales como estrategia para elevar el número de partos institucionales, que concierne a las estrategias sanitarias para reducir la mortalidad materna, mas no un medio para hacer más pertinente la atención del parto según la cultura de la comunidad.

Para los Matsigenka el parto no es sólo entendido como un evento biológico, es un suceso que contiene significancias sociales y culturales. Precisamente por ello tener conocimiento de las costumbres y prácticas durante el embarazo, parto y puerperio es indispensable para una mejor atención en el establecimiento de salud.

Las mujeres han mencionado, como parte de sus preferencias actuales en el parto, que el corte del cordón umbilical sea luego de que la placenta haya salido y que ésta sea entregada para hacer su entierro. Ambas acciones resultan no negociables, son imprescindibles para mantener un equilibrio en la salud del nuevo integrante del grupo familiar. Aunque la realización de estas prácticas culturales se redefinen con cada nuevo personal en el establecimiento.

Lo que antiguamente era un evento familiar, en casa y mayoritariamente femenino, ahora las mujeres han optado dar a luz en el establecimiento de salud como prevención a una complicación durante el parto en las casas, sin embargo prefieren el personal de salud mujer y la participación de la partera y familiares. La vestimenta, la posición de la gestante durante el parto y las dietas posparto que antiguamente era una regla de conducta de las mujeres expresada en la censura social, sobre todo de las mujeres mayores, son ahora consideradas como una elección para las mujeres, su derecho a decidir.

Como se ha querido demostrar, el conocimiento de las costumbres y prácticas en torno al parto-nacimiento no sólo son importantes para una mejor atención en los servicios de salud, con pertinencia cultural, sino que son valiosos en la construcción de la identidad como pueblo Matsigenka y en el fortalecimiento y reconocimiento del saber de las mujeres indígenas.

\section{Referencias bibliográficas}

AYNI Desarrollo (2016). Diagnóstico nutricional de niñas $y$ niños menores de 5 años de 3 comunidades nativas del distrito de Megantoni. La Convención, Cusco, Perú.

Baer, Gerhard (1994). Cosmología y shamanismo de los Matsiguenga (Perú oriental). Quito, Ecuador: Abya-Yala.

Bant, Astrid y Angélica Motta (2001). Escuchando a las mujeres de San Martín y Ucayali. Género y salud reproductiva. Lima, Perú: Movimiento Manuela Ramos.

CARE-Perú (2002). Percepciones y prácticas de salud en la Amazonía. Investigación participativa con comunidades Kichwas y Aguarunas. Lima, Perú: CARE-Perú. Programa Frontera Selva.

Encinas, Alfredo (2008). Historia de la Provincia de la Convención. Tomo II. Historia Social y Religiosa del Siglo $X X$. Lima, Perú: Centro Cultural José Pío Aza.

Fuller, Norma (2009). Relaciones de Género en la Sociedad Awajún. Lima, Perú: CARE Perú.

Instituto Lingüístico de Verano (2011). Diccionario Matsigenka - castellano. Serie lingüistica peruana No 56. Lima, Perú: Instituto Lingüístico de Verano.

Izquierdo, Carolina (2001). Betwixt and Between: Seeking Cure and Meaning Among the Matsigenka of the Peruvian Amazon (Tesis doctoral). University of California, Los Angeles, United States.

Izquierdo, Carolina y Glenn H., Jr. Shepard (2003). Matsigenka. En: Ember, C. R. y Ember, M. (Ed.), Encyclopedia of Medical Anthropology: Health and Illness in the World's Cultures, Vol. 2. (pp. 812-827). New York: Kluwer Academic/Plenum.

Medina, Armando y Julio Mayca (2006). Creencias y costumbres relacionadas con el embarazo, parto y 
puerperio en comunidades nativas Awajun y Wampis. Rev. Perú. med. exp. salud pública, 23(1), 22-32.

Ministerio de Salud (2000). Aspectos socioculturales y necesidades de salud reproductiva en comunidades nativas: estudio de investigación. Lima, Perú: Ministerio de Salud. Ministerio de Salud (1999). Salvarse con bien. El parto de la vida en los Andes y Amazonía del Perú (técnicas tradicionales de la atención del parto y el recién nacido). Lima, Perú: Ministerio de Salud, Proyecto 2000.

Norma Técnica de Salud 518. Diario Oficial El Peruano. Lima, Perú 21 de julio de 2016.

NureÑA, César (2009). Incorporación del enfoque intercultural en el sistema de salud peruano: la atención del parto vertical. Rev Panam Salud Pública, 26(4), 368-76.

PROCESO de desarrollo de la Comunidad de Camisea. s.f. Fundación ProNaturaleza.

Rrvas, Roxani (1994). La Mujer Cocama de Bajo Ucayali Matrimonio, Embarazo, Parto y Salud. Amazonía Peruana 24, 227-242.

SAlud Sin Límites Perú (2009). Cerrando brechas, aproximando culturas. Un modelo de atención maternoperinatal. Lima, Perú.

SAlud Sin Límites Perú (2008). Madre nativa: Experiencias acerca de la salud materna en las comunidades ashaninkas y nomatsiguengas. Lima, Perú.
Shepard, Glenn H., Jr. (2002). Three Days for Weeping: Dreams, Emotions, and Death in the Peruvian Amazon. Medical Anthropology Quarterly, New Series, 16(2), 200-229.

Vargas, Rossana y Paola Naccarato (1995). Allá, las antiguas eran parteras. Lima, Perú: Centro de la Mujer Peruana Flora Tristán.

Vela, Alejandro (2011). Conocimientos, actitudes y prácticas en salud de la población mashiguenga del Bajo Urubamba. Cuzco, Perú. Recuperado de http:// www.monografias.com/trabajos $88 /$ conocimientosactitudes-y-practicas-salud-mashiguenga-urubambacuzco-peru/conocimientos-actitudes-y-practicas-saludmashiguenga-urubamba-cuzco-peru.shtml

Velarde, Luz (2010). "Las parteras si saben, son importantes, sólo que están olvidadas». Situación actual de las parteras en los Andes del Sur del Perú (Ayacucho). Lima, Perú: Asamblea Nacional de Rectores.

Villavicencio, Nydia (2002). Hablan las mujeres de Ucayali. Género y salud reproductiva. Lima, Perú: Movimiento Manuela Ramos.

Zevallos, Arely (2010). Parto y poder. Los Harakmbut de San Miguel de Shintuya (tesis de maestría). Pontificia Universidad Católica del Perú. Escuela de Posgrado, Lima, Perú. 